\title{
Cerebrospinal Fluid Flow Cytometry: Utility in Central Nervous System Lymphoma Diagnosis
}

\author{
Ka Loong Kelvin Au, Sarah Latonas, Afshin Shameli, Iwona Auer, Christopher Hahn
}

\begin{abstract}
Background: Flow cytometry of the cerebrospinal fluid (CSF) is used in isolation or as an adjunct to cytology to increase the sensitivity of detecting central nervous system (CNS) lymphoma. We aimed to evaluate the sensitivity of CSF flow cytometry as a diagnostic screening tool for primary CNS lymphoma in patients presenting with undifferentiated neurologic symptoms. Methods: We retrospectively reviewed all CSF samples received by the Calgary Laboratory Services Flow Cytometry Laboratory from 2012 to 2015. Clinical data, laboratory investigations, radiologic imaging studies, and pathological data were analyzed. Clinical review extended to 2 years post-CSF flow cytometric testing. Results: Only 43/763 (5.6\%) samples of CSF flow cytometry in 28/573 (4.9\%) patients were found to be positive for a hematological malignancy in patients with undifferentiated neurologic symptoms. The overall sensitivity of the test was $13.8 \%$ with 25 patients with negative CSF flow cytometry later having a positive biopsy for CNS lymphoma. CSF flow cytometry was negative in all cases when at the time of CSF examination the patient did not have a previous hematological malignancy or findings of abnormal enhancement on MRI $(n=249)$. Conclusion: CSF flow cytometry has low utility in screening for primary CNS lymphoma in the absence of a previous history of hematologic malignancy or findings of abnormal enhancement on MRI.
\end{abstract}

RÉSUMÉ : Utilité de la cytométrie en flux du liquide cérébrospinal dans le diagnostic des lymphomes du système nerveux central. Contexte : La cytométrie en flux (CMF) du liquide cérébrospinal (LCS) est utilisée de façon isolée ou à titre de complément à la cytologie afin d'augmenter la sensibilité de détection des lymphomes du système nerveux central. Dans cette étude, nous voulons évaluer la sensibilité de la CMF du LCS comme outil de dépistage dans le cas de lymphomes primaires du système nerveux central dont sont atteints des patients donnant à voir des symptômes neurologiques indifférenciés. Méthodes : Nous avons passé en revue de façon rétrospective des échantillons de LCS reçus entre 2012 et 2015 par le Calgary Laboratory Services Flow Cytometry laboratory. Nous avons alors analysé les données cliniques disponibles, les recherches faites en laboratoire, les études d'imagerie radiologique ainsi que d'autres données de nature pathologique. Nous avons également effectué une analyse clinique deux ans après que des analyses de CMF du LCS ont été complétées. Résultats : Chez 28 patients sur 573 (4,9\%) aux prises avec des symptômes neurologiques indifférenciés, seuls 43 échantillons sur $763(5,6 \%)$ ayant fait l'objet d'analyses de CMF du LCS ont été jugés positifs pour une malignité hématologique. La sensibilité générale du test était de $13,8 \%$. Mentionnons aussi que 25 patients montrant des résultats négatifs à la CMF du LCS ont par la suite reçu des résultats positifs à une biopsie pour un lymphome du système nerveux central. Les résultats de CMF se sont par ailleurs révélés négatifs pour tous les patients chez qui, au moment des tests, on n'avait pas détecté une malignité hématologique antérieure ou chez qui on n'aurait pas détecté par IRM une amélioration anormale des agents de contraste $(\mathrm{n}=249)$. Conclusion : En l'absence d'antécédents de malignité hématologique ou de résultats prouvant par IRM une amélioration anormale des agents de contraste, la CMF du LCS a donc une faible utilité en matière de dépistage de lymphomes primaires du système nerveux central.

Keywords: Lymphoma, Cerebrospinal fluid, General neurology, Neurological practice, Neuro-oncology, Flow cytometry

doi:10.1017/cjn.2020.22

Can J Neurol Sci. 2020; 47: 382-388

\section{INTRODUCTION}

Central nervous system (CNS) lymphoma, though uncommon, can occur as a primary diagnosis or secondary from widespread systemic disease. Treatment of CNS lymphoma, including intrathecal chemotherapy and CNS irradiation, is not without its side effects; thus, it is imperative to make an accurate diagnosis. ${ }^{1}$ In newly diagnosed primary CNS lymphoma, delay in initiation of treatment was related to adverse outcome. ${ }^{2}$
The gold standard for diagnosis is brain biopsy by resection or stereotactic biopsy. Supportive testing includes radiologic evaluation, analysis of vitreous aspirate in patients with ocular involvement, and cerebrospinal fluid (CSF) analysis, including cytology and flow cytometry. ${ }^{3}$

Increasingly, flow cytometry in the CSF has been used in isolation or as an adjunct to cytology to increase the sensitivity of detecting CNS lymphoma. The sensitivity of CSF cytology

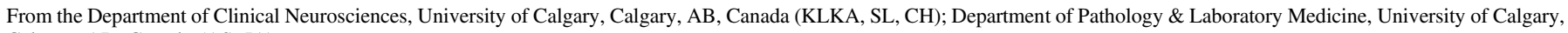
Calgary, AB, Canada (AS, IA)

Received November 22, 2019. Final Revisions Submitted January 22, 2020. Date of Acceptance January 25, 2020.

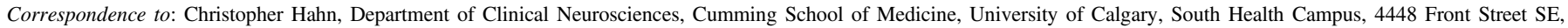

Calgary, AB T3M 1M4, Canada. Email: christopher.hahn@ahs.ca 
ranges from $2 \%$ to $32 \%{ }^{4}$ Poor sensitivity results from multiple causes including small sample size, delays in processing, sampling after exposure to corticosteroids, or inability to sample near the anatomic location of the lymphoma., Although the same sample limitations pertain to flow cytometric analysis, when combined with flow cytometry, up to $80 \%$ of lymphoma cases with CNS involvement are reported to be detected in the first CSF sample. ${ }^{6}$ In addition to this, flow cytometry has been reported to detect a larger amount of occult leptomeningeal disease in newly diagnosed aggressive B-cell lymphomas (11/51 or $22 \%$ ) compared to conventional cytology (1/51 or $2 \%){ }^{7}$

In a systematic review analyzing flow cytometry and cytology in patients with suspected meningeal involvement of lymphoid neoplasms, almost all studies $(24 / 27$ or $89 \%)$ state that the addition of flow cytometry to conventional cytology increases the diagnostic yield and positive cases of CNS involvement of lymphoma. ${ }^{8}$ Thus, CSF analysis with flow cytometry has become included in standard practice when investigating for CNS lymphoma involvement. ${ }^{9}$

The clinical presentation of CNS lymphoma may involve focal neurological deficits, which can be variable depending on the anatomical localization of the tumor. However, neurocognitive symptoms are the most common clinical presentation. ${ }^{3}$ Because of this, CNS lymphoma is often considered on the differential diagnosis of a patient presenting with nonspecific encephalopathy, even without prior clinical suspicion of a hematologic malignancy.

The benefit of investigating for CNS lymphoma with CSF flow cytometry on initial diagnostic evaluation includes patient comfort (fewer repeat lumbar punctures) and the possibility of a faster diagnosis. However, there have been several reports investigating the uncertain utility of CSF flow cytometry as a screening tool for patients presenting with undifferentiated altered mental status, and those without a prior clinical suspicion of a hematologic malignancy. 5,10

We aimed to investigate the diagnostic utility and usefulness of CSF flow cytometry at our institution by reviewing samples sent from all patients including those with undifferentiated altered mental status. This investigation has previously been shown to have a low-diagnostic yield in patients without a clinical suspicion of a hematological malignancy, and we hypothesized our study would support these findings in a large number of CSF samples. We suspected that in all patients with neurologic symptoms with suspicion of CNS involvement of a hematopoietic malignancy, the diagnostic yield of CSF flow cytometry is low. We hypothesized that the yield is so low that we would utilize these results to make a recommendation that it should not be included in first-line diagnostic testing in these patients.

In addition to this, we sought to identify additional diagnostic variables that may correlate with a positive flow cytometry result such as correlation with CSF cytopathology. Identifying diagnostic predictors may help increase the yield of CSF flow cytometry.

\section{Methods}

The study protocol was reviewed and approved by the Health Research Ethics Board of Alberta Cancer Committee. A retrospective review of all CSF flow cytometry samples sent to the University of Calgary Department of Pathology \& Laboratory
Medicine between January 1, 2012 and December 31, 2015. During this time period, 928 CSF samples were sent for CSF flow cytometry. Of 928 cases, 165 were excluded from review due to either patients being out of province (and thus no reliable followup clinical data) or they had a new diagnosis of acute leukemia (where CSF flow cytometry is performed routinely as part of the staging process), leaving 763 cases for review, as shown in Figure 1.

Clinical data including patient age, reason for CSF analysis, CSF tests including cell count, final clinical and pathologic diagnosis, medical history, previous immunosuppression, neurologic examination, history of previous hematopoietic malignancy, laboratory investigations, radiologic imaging studies, and subsequent clinical management were obtained. These findings were compared to the results of the CSF flow cytometry. Radiologic imaging studies were considered as baseline imaging if they were performed within 2 months of the CSF flow cytometry sample being sent. Clinical review extended to 2 years post-CSF flow cytometry testing to allow adequate time to capture potential new diagnosis of a hematopoietic malignancy via clinical follow-up.

\section{RESUlts}

We reviewed 763 cases of CSF flow cytometry taken from 573 patients. Table 1 shows predictive values of various clinical, radiographic, and pathology findings at the time of CSF flow cytometry collection. Of the 763 cases of CSF flow cytometry sent, 338/763 (44.3\%) samples had a prior hematologic malignancy known at the time of CSF collection for flow cytometry analysis. Of 763 (34.2\%) cases, 267 had abnormal enhancement found on their MRI brain or spine, 305/763 (40.0\%) did not have abnormal enhancement, and 191/763 (25.0\%) did not have MRI.

Of the 558 CSF samples with clinical data available, 114/558 $(20.4 \%)$ presented with altered level of consciousness or encephalopathy as the indication for CSF flow cytometry. This was the most common clinical presentation, followed by cranial nerve palsy in $65 / 558(11.6 \%)$, peripheral neuropathy in $55 / 558$ $(9.9 \%)$, cerebellar ataxia in $50 / 558(9.0 \%)$, and seizures in $49 / 558$ $(8.8 \%)$.

A positive result was more likely to occur in patients with a prior history of a hematological malignancy or abnormal enhancement on MRI $(p<0.0001)$. Of 763 cases, $43(5.6 \%)$ had CSF flow cytometry positive for a CNS hematopoietic malignancy in $28 / 573(4.9 \%)$ patients. Of the 43 positive samples, 41 had a previously known hematopoietic malignancy. One of these remaining two cases presented with acute obstructive hydrocephalus with strong irregular ependymal enhancement throughout the ventricular system on MRI. The patient underwent emergent external ventricular drain placement in the operating room. During this procedure, CSF flow cytometry was taken concurrently with a biopsy of the ependymal enhancement, both of which were positive for CNS lymphoma. The second case was a patient who had a prior history of a double lung transplant presenting with headache, fever, confusion, and ataxia. There was diffuse leptomeningeal enhancement on MRI. CSF flow cytometry in this patient revealed a clonal B-cell population that was diagnosed as Epstein-Barr virus (EBV) positive post-transplant lymphoproliferative disorder in addition to having high titers of EBV in both serum and CSF. Thus, all positive CSF flow 
Table 1: Predictive values for CSF flow cytometry cases

\begin{tabular}{|c|c|c|c|c|c|c|}
\hline \multirow{2}{*}{ Item } & \multicolumn{3}{|c|}{ Positive cases $(n=43)$} & \multicolumn{3}{|c|}{ Negative cases $(n=720)$} \\
\hline & Present & Not present & Unknown & Present & Not present & Unknown \\
\hline Abnormal enhancement on MRI & 26 & 9 & 8 & 241 & 296 & 183 \\
\hline $\begin{array}{l}\text { Previous history of any hematological } \\
\text { malignancy }\end{array}$ & 41 & 2 & 0 & 297 & 423 & 0 \\
\hline $\begin{array}{l}\text { Abnormal enhancement on MRI or previous } \\
\text { history of any hematological malignancy }\end{array}$ & 43 & 0 & 0 & 470 & 250 & 0 \\
\hline $\begin{array}{l}\text { Abnormal enhancement on MRI and previous } \\
\text { history of any hematological malignancy }\end{array}$ & 24 & 11 & 8 & 67 & 653 & 0 \\
\hline Elevated peripheral lymphocytes $(>3.310 \mathrm{E} 9 / \mathrm{L})$ & 11 & 27 & 5 & 35 & 595 & 90 \\
\hline Elevated CSF WBC (>10.0 10E6/L) & 14 & 19 & 10 & 102 & 525 & 93 \\
\hline Elevated CSF neutrophils $(>15 \%)$ & 1 & 32 & 10 & 24 & 603 & 93 \\
\hline Elevated CSF lymphocytes (>15\%) & 10 & 23 & 10 & 107 & 520 & 93 \\
\hline Elevated CSF atypical lymphoid cells $(>15 \%)$ & 5 & 28 & 10 & 2 & 625 & 93 \\
\hline Abnormal CSF cytopathology & 19 & 6 & 18 & 26 & 441 & 253 \\
\hline Abnormal neurological examination & 36 & 1 & 6 & 41 & 517 & 162 \\
\hline
\end{tabular}

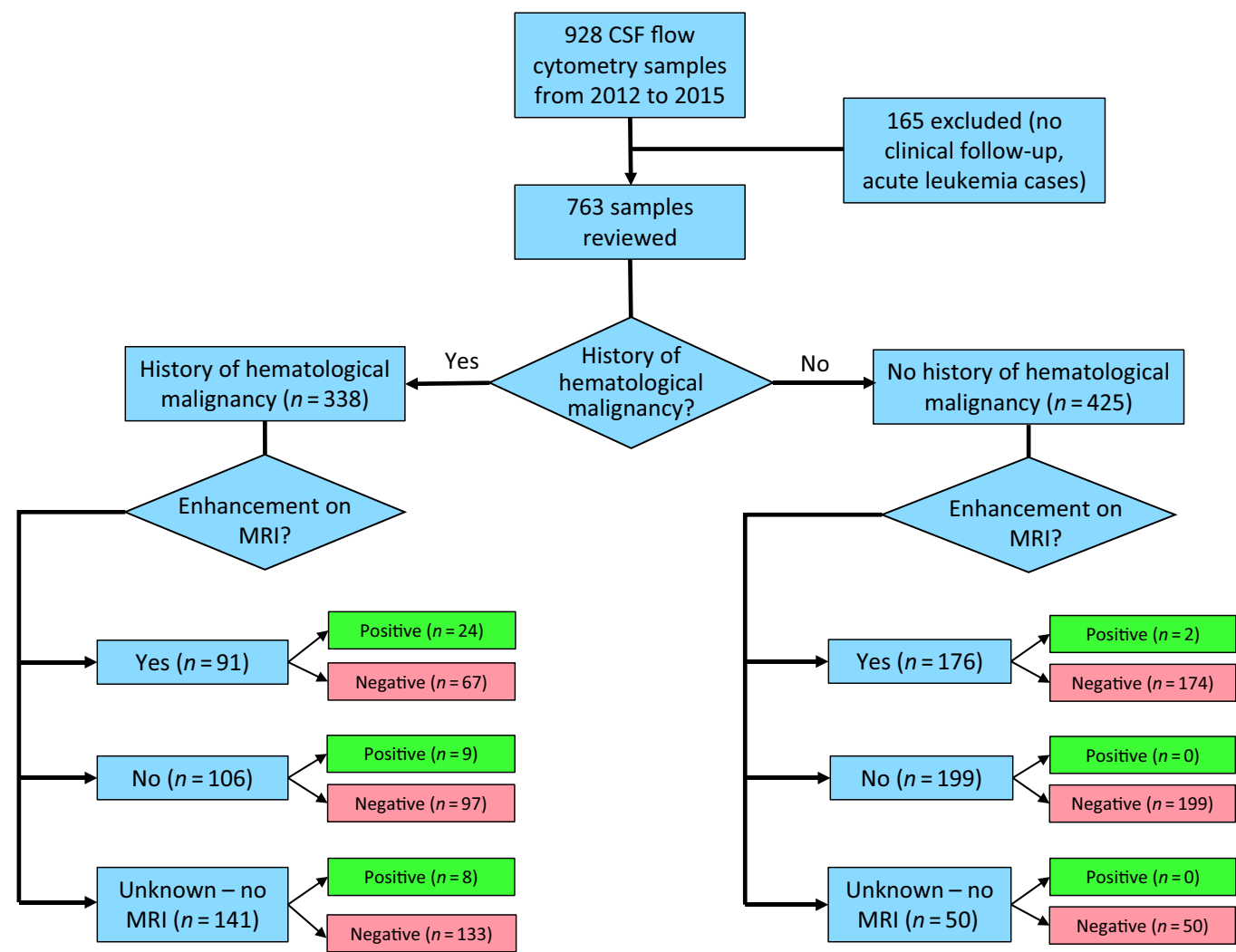

Figure 1: Flow diagram of CSF flow cytometry samples.

cytometry cases either had a previously known hematopoietic malignancy or abnormal enhancement on MRI suspicious for CNS lymphoma prior to sending the CSF for sampling.

There were 425 CSF flow cytometry samples without a prior known history of a hematological malignancy, 176 of which had abnormal enhancement on MRI and the remaining 249 did not.
Only the aforementioned 2/176 (1.1\%) cases with abnormal enhancement on MRI but no history of hematological malignancy had positive CSF flow cytometry. None of the 249 samples without a prior history of a hematological malignancy nor abnormal enhancement on MRI were positive for flow cytometry (Figure 1). However, 19/249 (7.6\%) cases went on to have CNS 
Table 2: Imaging and biopsy characteristics

\begin{tabular}{|c|c|c|c|c|c|c|}
\hline \multirow{2}{*}{ Item } & \multicolumn{3}{|c|}{ Positive cases $(n=43)$} & \multicolumn{3}{|c|}{ Negative cases $(n=720)$} \\
\hline & Present & Not present & Unknown & Present & Not present & Unknown \\
\hline Lymphadenopathy on CT chest/abdo/pelvis & 11 & 17 & 15 & 80 & 324 & 316 \\
\hline Splenomegaly on CT chest/abdo/pelvis & 12 & 16 & 15 & 49 & 355 & 316 \\
\hline Enhancement on CT head & 6 & 23 & 14 & 12 & 349 & 359 \\
\hline Evidence of active disease on FDG/PET & 7 & 0 & 36 & 48 & 83 & 589 \\
\hline Abnormal bone marrow biopsy & 8 & 0 & 35 & 44 & 34 & 642 \\
\hline Lymphoma on lymph node biopsy & 3 & 0 & 40 & 12 & 1 & 707 \\
\hline Lymphoma on brain biopsy & 4 & 0 & 39 & 25 & 17 & 678 \\
\hline
\end{tabular}

biopsy of which 6/19 were positive for diffuse large B-cell lymphoma (DLBCL).

\section{Brain Biopsy}

Biopsy and imaging characteristics of the CSF flow cytometry cases can be found in Table 2. Forty-two patients with negative CSF flow cytometry went on to have CNS biopsy. Of these 42 patients, 25 (59.5\%) had biopsies which led to a diagnosis of CNS lymphoma via subsequent biopsy. Included in these 25 patients are the aforementioned 6 patients who had CNS lymphoma diagnosed via biopsy without a history of a hematological malignancy nor did they have abnormal enhancement on MRI. Of these 25, $10(40.0 \%)$ patients had a diagnosis of primary CNS lymphoma, whereas 15/ $25(60.0 \%)$ had CNS involvement of systemic lymphoma. Of these 25 patients, several had repeated CSF flow cytometry samples. Three of the patients had their CSF flow cytometry repeated twice, and three patients had their CSF flow cytometry repeated three or more times, all with negative results prior to biopsy. The remaining $17 / 42$ patients with negative CSF flow cytometry who had biopsy showed results other than CNS lymphoma. The results of these brain biopsies included neurosarcoidosis (2), viral encephalitis (2), inflammatory demyelination (4), metastases (1), high-grade glioma (3), vascular malformation (2), inconclusive (1), and biopsy data not available (2).

Only four positive CSF flow cytometry samples in four separate patients went on to have confirmatory brain biopsy, which all showed the hematopoietic malignancy suspected on flow cytometry. The remaining 39 samples which had positive CSF flow cytometry (performed in the remaining 24 patients) did not have a brain biopsy.

\section{Serial CSF Examination}

There were 54 patients who had 2 CSF samples taken for CSF flow cytometry (108 CSF flow cytometry samples), and 39 patients who had 3 or more CSF samples (175 CSF flow cytometry cases). Of these, $3 / 54$ (5.6\%) patients showed positive CSF flow cytometry on the second CSF examination. CSF flow cytometry was positive on the third or greater examination in $5 / 39(12.8 \%)$ of patients.

\section{Cytopathology}

There was a cytomorphological correlation to the CSF flow cytometry; of the 43 cases with positive CSF flow cytometry, 19/43 (44.2\%) samples had associated abnormal CSF cytology (ranging from malignant cells to atypical cells), 6/43 (14.0\%) were negative, and 18/43 (41.9\%) were unknown (i.e., no cytopathology). Of the 720 negative CSF flow samples, 26/720 (3.6\%) had abnormal CSF cytology, 441/720 (61.3\%) were negative, and $253 / 720(35.1 \%)$ were unknown. Of the 25 samples with negative CSF flow cytometry that went on to have a diagnosis of CNS lymphoma on biopsy, 3 (12.0\%) had abnormal cytopathology on CSF testing.

\section{Discussion}

In this relatively large series of consecutive CSF samples, we confirm that the yield of CSF flow cytometry is low in patients with undifferentiated neurologic symptoms when compared against the gold standard of brain biopsy or longitudinal clinical follow-up for 2 years. No patient without a previous history of hematologic malignancy or findings of abnormal enhancement on MRI had positive CSF flow cytometry.

The relatively high number (25) of patients with negative CSF flow cytometry that went on to be diagnosed with CNS lymphoma via subsequent biopsy demonstrates the lack of overall sensitivity $(13.8 \%)$ of the test, which further argues against it being used as a first-line screening test for CNS lymphoma in patients with no clinical or radiological suspicion. In our study, 8 patients from the 720 cases of negative CSF flow cytometry went on to have CNS lymphoma diagnosed via serial CSF flow cytometry examination. Apart from these cases, all patients underwent clinical chart review which extended 2 years postCSF analysis and no further clinical or laboratory diagnoses of CNS lymphoma occurred, making it unlikely the remaining negative CSF flow cases were false negatives. This supports previous work suggesting limited utility of CSF flow cytometry for the diagnosis of CNS lymphoma outside of patients with a previous history of hematologic malignancy or suspicious radiologic findings. $5,10-12$

In Pittman et al., CSF analysis of 102 patients who had no history of lymphoma and no suspicious radiologic findings revealed that $0 / 102(0 \%)$ had positive CSF flow cytometry. ${ }^{5}$ Only one patient from this group $(0.8 \%)$ was later diagnosed with primary CNS lymphoma on tissue histology after two negative CSF flow cytometry and one negative cytology. ${ }^{5}$ Other reports corroborate these findings, showing only positive flow cytometry in $1 / 71(1.4 \%),{ }^{11} 1 / 18(5.6 \%),{ }^{12}$ and $1 / 118(0.8 \%)^{10}$ of samples collected from patients without history of hematolymphoid malignancy. These patients had various undifferentiated 


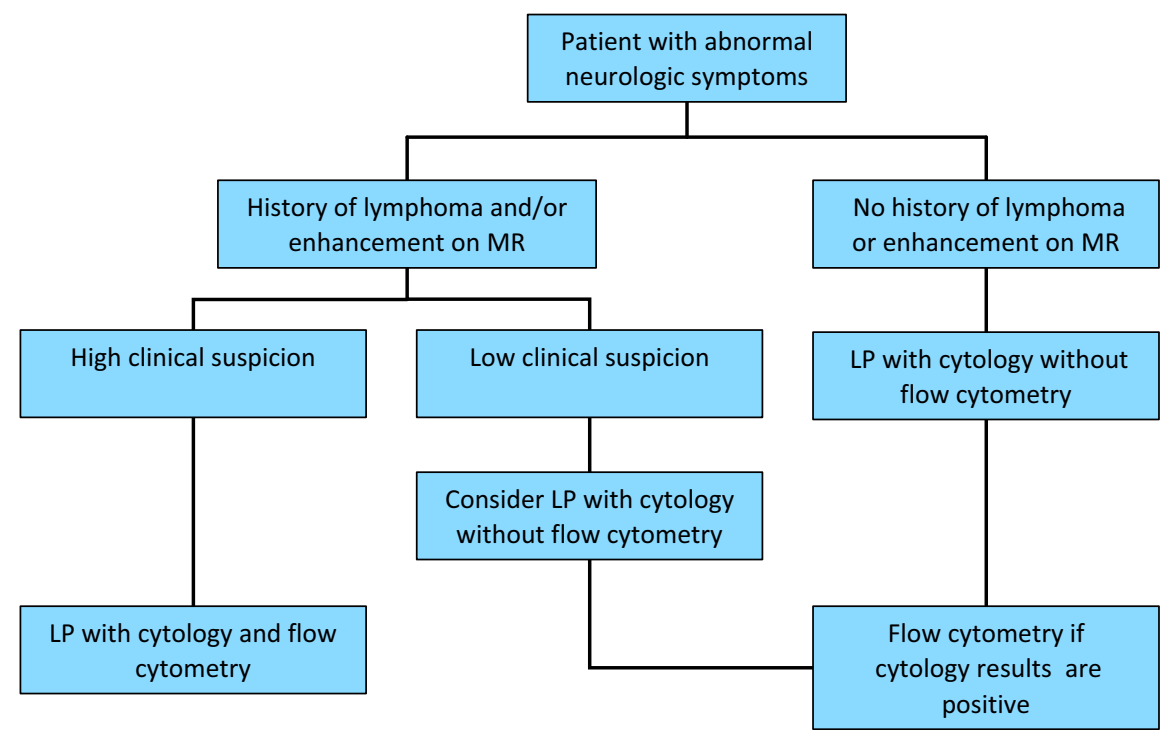

Figure 2: Proposed algorithm of CSF flow cytometry testing. Adapted from Pittman et al. ${ }^{5}$, used with permission.

neurological symptoms listed as indications for the CSF flow cytometry in $35 / 71(49.3 \%),{ }^{11} 118 / 118(100.0 \%),{ }^{10}$ without prior history of hematological malignancy. The number of patients with undifferentiated neurological symptoms without prior hematological malignancy was not indicated specifically in the other two reports, ${ }^{5,12}$ but 291/373 (78\%) of all samples had undifferentiated neurological symptoms in Pittman et al. ${ }^{5}$

Prior authors have proposed a triage algorithm where CSF flow cytometry and cytology are only utilized when certain clinical criteria are met to employ rational and appropriate use of laboratory services. ${ }^{5}$ Some suggest only utilizing flow cytometry when there is a history of a hematologic malignancy, or when there are atypical cells found on cytology that are suspicious or worrisome for a hematologic malignancy. ${ }^{13}$

Our findings support this algorithm in that there was a cytopathological correlation with atypical lymphoid, atypical and malignant cells found on cytopathology corresponding to a higher likelihood of a positive CSF flow cytometry result. A negative (i.e., normal) CSF cytology would make the likelihood of the CSF flow cytometry being positive less likely. Thus, we suggest that adopting a similar testing algorithm that includes taking into account a prior hematological malignancy, abnormal enhancement on MRI, and abnormal cells on cytopathology would increase the diagnostic yield of CSF flow cytometry (Figure 2).

One of the common criticisms of such an approach is that sequential testing of CSF is that this would lead to multiple lumbar punctures and thus discomfort for the patient. We understand that this algorithm may be difficult to implement at the clinician level due to this reason. However, there are also challenges with having the receiving laboratory adopt the algorithm. CSF samples for flow cytometry typically must be performed within $24 \mathrm{~h}$ of CSF collection in order to yield accurate results. CSF cytology often takes longer than this to be reported and, thus, holding CSF fluid for potential CSF flow cytometry testing is not feasible. However, if the CSF cytology is abnormal, performing another lumbar puncture for CSF flow cytometry is still less invasive than a brain biopsy to confirm the diagnosis of CNS lymphoma, so repeated lumbar punctures in low risk patients is likely justified. The exact best method to adopt this algorithm for CSF flow cytometry testing is yet to be determined and its implementation may be topic for further studies.

Current guidelines recommend that the diagnosis of CNS lymphoma not be made on the basis of CSF flow cytometry alone. The test is meant to be used in conjunction with clinical, imaging, and ideally pathological data to make the diagnosis. ${ }^{14}$ Definitive tissue histopathological diagnosis of CNS lymphoma is preferred prior to initiating chemotherapy given the uncertain specificity and sensitivity of CSF flow cytometry and the need to assess cytomorphology and pattern of tissue involvement by histologic exam. There are instances, however, of when CSF flow cytometry can be the preferred test for diagnosis of CNS lymphoma and we continue to acknowledge its usefulness in the correct clinical context. CSF flow cytometry may be used alone in the absence of a mass that can be biopsied when suspicion of lymphoma is high or in the instance where the mass is in a location where biopsy has a high-surgical risk. In these circumstances, the positive CSF flow cytometry result along with the clinical signs and symptoms may guide the clinician to making the diagnosis of CNS lymphoma and beginning treatment. That being said, the standard diagnostic approach for primary CNS lymphoma remains stereotactic needle brain biopsy when possible. ${ }^{15}$

On review of our cohort, there were six patients who had negative CSF flow cytometry, absence of enhancing lesions on MRI, and no prior history of a hematopoietic malignancy that went on to have brain biopsy revealing the diagnosis of DLBCL. All six patients had a non-enhancing abnormality on brain MRI or CT. After negative workup, a brain biopsy was pursued to make the diagnosis which was DLBCL in all cases. Two of these cases actually had initial treatment with dexamethasone, leading to initial improvement of their symptoms and imaging abnormalities. However, upon taper of the steroid, they returned with worsened symptoms and imaging abnormalities. Brain biopsy was pursued and led to the diagnosis of DLBCL. Although by 
following our triage algorithm these patients would not have had CSF flow cytometry sent, it is possible that even with a positive CSF flow cytometry result these patients may have still undergone brain biopsy prior to committing them to a therapeutic course of chemotherapy. As mentioned, confirmation with tissue biopsy is the gold standard for diagnosis of and treatment planning for primary CNS lymphoma. ${ }^{14,15}$

Others have reviewed serial CSF examinations in diagnosing hematological disease, but have found the addition of repeated CSF flow cytometry in succession is of low yield. ${ }^{16}$ In this study, 477/613 patients had 2 CSF examinations and 136/613 patients had 3 or greater CSF examinations. Of the 477 patients with two CSF examinations, $2(0.4 \%)$ had a large B cell lymphoma diagnosed on the second CSF flow cytometry exclusively, but none with three or greater CSF examinations $(0 / 136)$ had a diagnosis of a CNS hematological malignancy found on CSF flow cytometry with subsequent CSF examination. Of these patients who had suspicion of hematological malignancy (but non-diagnostic) on serial CSF examinations, five underwent additional confirmatory tissue biopsy (including brain, bone marrow, and median nerve). ${ }^{16}$ In our study, out of 54 patients who had two CSF examinations, 3/ $54(5.6 \%)$ had positive CSF flow cytometry sample on the second CSF examination. Out of 39 patients who had three or greater CSF examinations, 5/39 (12.8\%) patients have their positive CSF flow cytometry sample on the third or greater CSF examination. In total, this made up 8/28 (28.6\%) of patients who had positive CSF flow cytometry in our study. Of note, however, all eight of these patients had a prior history of hematological malignancy prior to serial CSF examination. Although our yield of serial CSF examinations is higher compared to what is previously reported, all of the patients who had a diagnosis of CNS lymphoma from serial CSF examination came from patients with a prior hematological malignancy. Thus, we agree that serial CSF examination in patients without a prior hematological malignancy is low yield, as none of these patients had positive CSF flow cytometry on serial CSF examination. Alternatively, this potentially makes an argument for serial CSF examinations in only those patients who have a prior history of hematological malignancy. In addition to this, the impact of serial CSF sampling on time to diagnosis and therefore potential treatment delay versus patients who went on to CNS biopsy immediately are questions that future research may help clarify.

\section{Study Limitations}

Our study represents the example of one tertiary center for 4 years, which may not be reflective of all patients who have a suspicion of CNS lymphoma and thus may not be generalizable to all centers. Our retrospective approach is limited by, at times, incomplete or missing data. We are also limited by the arbitrary cutoff of following these patients' medical records for 2 years post-CSF flow cytometry testing, as patients could have potentially went on to be diagnosed with CNS lymphoma after this date. In addition to this, not all patients received all testing noted (imaging, pathology, laboratory testing) and where information was lacking we noted this by indicating the data were unknown.

Our cohort of patients and samples was obtained by generating a list of patients who had CSF flow cytometry performed. This would exclude patients who had a diagnosis of CNS lymphoma on biopsy alone without CSF analysis. The true specificity and sensitivity of CSF flow cytometry is unlikely to be determined from our study as a result. As mentioned, the gold standard test for the diagnosis of CNS lymphoma is tissue biopsy, which was not performed for all patients in our study, as often the risks of a confirmatory tissue biopsy procedure and delay in treatment exceeded the benefit. Thus, many of our patients obtained their diagnosis of CNS lymphoma based on their positive CSF flow cytometry taken together with the clinical history and examination in the absence of gold standard tissue diagnosis.

\section{Conclusion}

Our study supports previous findings suggesting that investigating for CNS lymphoma with CSF flow cytometry is most useful in a selective group of patients as opposed to as a screening tool for all patients that present with nonspecific neurologic symptoms. Our data have identified the two most important factors to consider prior to attempting CSF flow cytometry: determining whether the patient has had a prior history of a hematologic malignancy (CNS or systemic) or if they have abnormal enhancement on MR neuroimaging of the CNS. If neither of these factors are present, then our data suggest the yield of CSF flow cytometry to be very low. In addition to this, consideration of cytopathology results can also help increase the yield of CSF flow cytometry.

In a time where there is growing pressure to reduce overall health care costs, it becomes ever more important for clinicians to utilize tests such as flow cytometry only when clinically appropriate. The use of a triage algorithm as included herein could help prevent unnecessary testing in cases where CSF flow cytometry is unlikely to provide helpful diagnostic information in patients with nonspecific neurologic symptoms.

\section{DiscLOSURES}

The authors have no conflicts of interest to declare.

\section{STATEMENT OF AUTHORSHIP}

KLKA - Manuscript draft, data acquisition.

SL - Data acquisition, manuscript revision.

AS - Manuscript revision, acquisition, and interpretation of laboratory findings.

IA - Conception and design of study, manuscript revision.

$\mathrm{CH}$ - Study supervision, conception and design of study, data interpretation, and manuscript revision.

\section{REFERENCES}

1. Batchelor T, Thye L, Habermann T. Current management concepts: primary central nervous system lymphoma, natural killer T-cell lymphoma nasal type, and post-transplant lymphoproliferative disorder. Am Soc Clin Oncol Educ Book. 2016;35:e354-66.

2. Rubenstein JL, Hsi ED, Johnson JL, et al. Intensive chemotherapy and immunotherapy in patients with newly diagnosed primary CNS lymphoma: CALGB 50202 (alliance 50202). J Clin Oncol.. 2013;31(25):3061-68.

3. Paydas S. Primary central nervous system lymphoma: essential points in diagnosis and management. Med Oncol.. 2017;34(4):61.

4. Scott B, Douglas V, Tihan T, et al. A systematic approach to the diagnosis of suspected Central nervous system lymphoma. JAMA Neurol. 2013;70(3):311-19.

5. Pittman M, Treese S, Chen L, et al. Utility of flow cytometry of cerebrospinal fluid as a screening tool in the diagnosis of central nervous system lymphoma. Arch Pathol Lab Med. 2013; 137(11):1610-18. 
6. Bromberg JE, Breems DA, Kraan J, et al. CSF flow cytometry greatly improves diagnostic accuracy in CNS hematologic malignancies. Neurology. 2007;68(20):1674-79.

7. Hegde U, Filie A, Little R, et al. High incidence of occult leptomeningeal disease detected by flow cytometry in newly diagnosed aggressive B-cell lymphomas at risk for central nervous system involvement: the role of flow cytometry versus cytology. Blood. 2005;105(2):496-502.

8. Canovi S, Campioli D. Accuracy of flow cytometry and cytomorphology for the diagnosis of meningeal involvement in lymphoid neoplasms: a systematic review. Diagn Cytopathol. 2016;44(10):841-56.

9. Abrey LE, Batchelor TT, Ferreri AJ, et al. Report of an international workshop to standardize baseline evaluation and response criteria for primary CNS lymphoma. J Clin Oncol. 2005; 23(22):5034-43.

10. Kovach AE, DeLelys ME, Kelliher AS, et al. Diagnostic utility of cerebrospinal fluid flow cytometry in patients with and without prior hematologic malignancy. Am J Hematol. 2014;89(10):978-84.
11. Craig FE, Ohori NP, Gorrill TS, et al. Flow cytometric immunophenotyping of cerebrospinal fluid specimens. Am J Clin Pathol. 2011;135(1):22-34.

12. Roma AA, Garcia A, Avagnina A, et al. Lymphoid and myeloid neoplasms involving cerebrospinal fluid: comparison of morphologic examination and immunophenotyping by flow cytometry. Diagn Cytopathol. 2002;27(5):271-75.

13. Hsi ED. Flow cytometry in cerebrospinal fluid-rational use of laboratory services. Am J Hematol. 2014;89(10):941-42.

14. Fox CP, Phillips EH, Smith J, et al. Guidelines for the diagnosis and management of primary central nervous system diffuse large B-cell lymphoma. Br J Haematol. 2019;184(3): $348-63$.

15. Carnevale J, Rubenstein JL. The challenge of primary CNS lymphoma. Hematol Oncol Clin North Am. 2016;30(6): 1293-316.

16. Scharf EL, Hanson CA, Howard MT, et al. Serial cerebrospinal fluid examinations to diagnose hematological malignancy causing neurological disease. J Neurooncol. 2016;129(1):77-83. 